\title{
Skeletal Muscle Mass Indices in Healthy Young Mexican Adults Aged 20-40 Years: Implications for Diagnoses of Sarcopenia in the Elderly Population
}

\author{
H. Alemán-Mateo and Roxana E. Ruiz Valenzuela \\ Coordinación de Nutrición, Centro de Investigación en Alimentación y Desarrollo (CIAD), \\ A.C. Carretera a La Victoria Km. 0.6, 83304 Hermosillo, Sonora, Mexico \\ Correspondence should be addressed to H. Alemán-Mateo; helio@ciad.mx
}

Received 29 August 2013; Accepted 15 December 2013; Published 6 February 2014

Academic Editors: S. Hirsch and M. A. Kamimura

Copyright (C) 2014 H. Alemán-Mateo and R. E. Ruiz Valenzuela. This is an open access article distributed under the Creative Commons Attribution License, which permits unrestricted use, distribution, and reproduction in any medium, provided the original work is properly cited.

\begin{abstract}
Background and Objectives. Skeletal muscle and skeletal muscle indices in young adults from developing countries are sparse. Indices and the corresponding cut-off points can be a reference for diagnoses of sarcopenia. This study assessed skeletal muscle using dual-energy X-ray absorptiometry (DXA) in healthy male and female subjects aged 20-40 years and compared their appendicular skeletal muscle mass (ASM) and total-body skeletal muscle (TBSM) indices using certain cut-off points published in the literature. Methods. A sample of 216 healthy adults men and women from northwest Mexico was included. Body composition was assessed by DXA and several published DXA-derived skeletal muscle indices were compared. Results. Both, ASM and TBSM were higher in men compared to the women group $(23.0 \pm 3.4$ versus $15.9 \pm 1.6 \mathrm{~kg} ; P<0.05$ and $26.5 \pm 4.1$ versus $16.9 \pm 1.9 \mathrm{~kg} ; P<0.05$, resp.). These differences were also valid for both indices. When derived cut-off points were compared with the most reported indices, significant differences were found. Interpretation and Conclusion. Published cut-off points from Caucasians are higher than cut-off point derived in this sample of Mexican subjects. The new DXA-derived cut-off points for ASM proposed herein may improve diagnoses of sarcopenia in the geriatric Mexican population.
\end{abstract}

\section{Introduction}

Skeletal muscle (SM) plays a central role in many biological functions, such as movement and metabolism, so disruptions in this component of body composition can have a marketed influence on health and disease [1]. Also, we cannot ignore the influence of this factor on nutritional status and functionality at older ages. Skeletal muscle in both men and women subjects remains relatively constant during the third and fourth decades of life but begins to decline noticeably at $\sim 45$ years of age [2-4]. It has been suggested that the maintaining muscle mass in old age is a key factor for conserving physical capacity and enabling independent lifestyles in old age. Human skeletal muscle mass depends basically on stature, weight, age, and gender. In a published study these factors explained $>80 \%$ between-individual within-ethnic group
(African-Americans and Caucasians) differences in SM mass [3].

The loss of SM is a common problem in older adults from around the world that reaches a prevalence ranging from $7 \%$ to over $50 \%$ [5]. At one time, the age-related loss of skeletal muscle was called sarcopenia, but now its definition is not limited only to the loss of muscle mass. Sarcopenia comprised the loss both muscle mass and strength with a risk of adverse outcomes such as physical disability, poor quality of life, and even death [5]. In terms of diagnoses, sarcopenia is characterized by low muscle mass plus low muscle strength or low physical performance. To establish "low muscle mass", some researchers have assessed the total or appendicular skeletal muscle in young adults (the age range in which muscle reaches a plateau and then remains relatively constant). It is important to highlight that the 
absolute skeletal muscle mass $(\mathrm{kg})$ is normally converted to skeletal muscle indices. Baumgartner et al. [6] summed the muscle mass of the four limbs assessed by DXA and used the term appendicular skeletal muscle mass (ASM) to refer to this sum. They then defined the appendicular skeletal muscle mass index (ASMI) as ASM/height ${ }^{2}\left(\mathrm{~kg} / \mathrm{m}^{2}\right)$. ASMI twostandard deviations below the mean ASMI of healthy young male and female reference groups were defined as the genderspecific cut-off points for sarcopenia (original term used).

Several cut-off points based on the ASMI or totalbody SM mass index (TBSMI) have been derived [6-13]; however, these cut-off points depend on several factors: the measurement technique used, age range, sex, ethnicity, and the availability suitable references studies. For the Mexican case, unfortunately, no such reference SM data exist, so the few published studies on sarcopenia have used a cut-off point based on an ASMI derived from a young Caucasian population [14]; even though could well bias the prevalence of the loss of skeletal muscle or sarcopenia. In addition, several studies have shown significant differences in the prevalence of sarcopenia when different indices are used interchangeably or are applied to populations distinct from the original sex-specific group of subjects [15]. Due to these issues, the recommendation is that these indices would ideally refer to sex-specific ethnic group populations [5]. Therefore, this study assessed skeletal muscle using dualenergy X-ray absorptiometry in healthy male and female subjects aged 20-40 years and compared their ASM and TBSM indices using certain cut-off points published in the literature.

\section{Materials and Methods}

2.1. Study Design and Subjects. This is a cross-sectional, nonprobability study that included 216 apparently healthy adult men and women from northwest Mexico. All volunteers were selected on the bases of inclusion and exclusion criteria. All men and (nonpregnant) female subjects were between 20 and 40 years of age, were apparently healthy by self-report, and were either born in the state of Sonora, Mexico or had lived there for at least five years. Subjects were excluded if their body weight or height was outside the DXA bed dimensions. Subjects who reported changes in body weight in the last six months due primary to caloric restriction or increased physical activity were not included. For safety reason, pregnant women were not invited to participate and those who were positive on a pregnancy test (DIAGMEX commercial test) were not included for the DXA measurements. Also, volunteers who were taking medications that affect body composition and those with protein supplementation were excluded. Most volunteers had lifestyles that involved only light occupational and leisure time activities. Finally, trained technicians took all anthropometric and body composition measurements of subjects under fasting conditions or at least two hours after subject's most recent meal or drink.

\subsection{Anthropometry}

2.2.1. Body Weight. This variable was measured with subjects dressed in light clothing and shoes less (avoiding extra weight such as wallets, money, and phones). The Digital Electronic Scale (PV-150 K DNA, Japan) used was calibrated before taking measurements.

2.2.2. Height. This was measured without shoes and according to our standardized protocol, using a calibrated Holtain stadiometer (Holtain Ltd, Dyfed, UK).

2.2.3. Body Mass Index. BMI was calculated on the basis of body weight and height measurements and classified according to WHO's 1997 cut-off points [16].

2.3. Body Composition. The evaluation of body composition including the lean tissue was carried out under fasting conditions or at least two hours after the most recent light meal or drinks, following established guidelines and using a DXA (DPX-MD+; GE Lunar Madison, WI, USA). Regional body composition components were determined from the DXA scans, following the recommended anatomical landmarks. The sum of nonfat plus nonbone tissue in both arms and legs was used to represent ASM [17]. Limb skeletal muscle mass represents $75 \%$ of total body skeletal muscle [18]. Other body composition components, such as total body lean tissue and fat mass, were also assessed. From DXA-derived ASM, totalbody skeletal muscle mass was predicted using Kim's equation [19].

2.4. Skeletal Muscle Mass Indices and Cut-Off Points. Two indices were calculated: ASMI as ASM/height ${ }^{2}\left(\mathrm{~kg} / \mathrm{m}^{2}\right)$ and TBSMI as TBSM/height ${ }^{2}\left(\mathrm{~kg} / \mathrm{m}^{2}\right)$. Two cut-off points were established considering two standard deviations below the mean of both indices derived from the SM assessed in a sample of healthy young man and women from northwest Mexico.

\subsection{Skeletal Muscle Mass Indices and Cut-Off Points Derived} from the Other Populations. For this study, Baumgartner et al., Sanada et al., Lau et al., Wen et al., and Kim et al's sexspecific cut-off points for ASMI [6-10] and Lau et al., Tichet et al., Chien et al., and Masanes et al. [8,11-13] sex-specific cutoff points for TBSMI were used for purposes of comparison.

2.6. Statistical Analyses. Results are presented as mean standard deviations by sex, while significant differences between man and women were tested by a two-sample $t$-test. A onesample $t$-test was also used to test the significant differences between our sex-specific cut-off points compared to the different published ASMI and TBSMI cut-off points derived from a young adult ethnic group populations.

\section{Results}

This study included 216 subjects, $63 \%$ were men and the rest women. Average was $27.6 \pm 5.2$ years, with a range of 18 to 40 years. The mean BMI was $24.7 \mathrm{~kg} / \mathrm{m}^{2}$ which falls into the "normal" range according to the WHO's classification. Regarding the anthropometric data collected, a significant 
TABLE 1: Anthropometry and body composition components of healthy young Mexican adults.

\begin{tabular}{|c|c|c|c|}
\hline Variables & $\operatorname{Men}(n=136)$ & Women $(n=80)$ & Both men and women \\
\hline Age, (years) & $27.3 \pm 5.0$ & $28.2 \pm 5.6$ & $27.6 \pm 5.2$ \\
\hline Weight, (kg) & $77.9 \pm 13.0$ & $60.4 \pm 8.5^{*}$ & $71.5 \pm 14.3$ \\
\hline Height, (m) & $1.74 \pm 0.1$ & $1.61 \pm 0.04^{*}$ & $1.69 \pm 0.08$ \\
\hline BMI, $\left(\mathrm{kg} / \mathrm{m}^{2}\right)$ & $25.7 \pm 3.6$ & $23.2 \pm 3.1^{*}$ & $24.7 \pm 3.6$ \\
\hline ASM, (kg) & $23.0 \pm 3.4$ & $15.9 \pm 1.6^{*}$ & $20.1 \pm 4.7$ \\
\hline ASMI, $\left(\mathrm{kg} / \mathrm{m}^{2}\right)$ & $7.5 \pm 0.8$ & $5.8 \pm 0.5^{*}$ & $6.6 \pm 1.1$ \\
\hline TBSM, (kg) & $26.5 \pm 4.1$ & $16.9 \pm 1.9^{*}$ & $22.9 \pm 5.7$ \\
\hline TBSMI, $\left(\mathrm{kg} / \mathrm{m}^{2}\right)$ & $8.6 \pm 1.0$ & $6.5 \pm 0.6^{*}$ & $7.8 \pm 1.3$ \\
\hline FFM, (kg) & $52.9 \pm 7.1$ & $34.2 \pm 3.2^{*}$ & $46.0 \pm 10.8$ \\
\hline Fat mass, (kg) & $20.5 \pm 8.5$ & $22.2 \pm 6.6^{* *}$ & $21.2 \pm 4.9$ \\
\hline Truncal fat, (kg) & $13.1 \pm 5.4$ & $11.3 \pm 3.8^{* *}$ & $12.4 \pm 7.9$ \\
\hline
\end{tabular}

BMI: body mass index; ASM: appendicular skeletal muscle mass; ASMI: appendicular skeletal muscle mass index; TBSM: total-body skeletal muscle mass; TBSMI: total-body skeletal muscle mass index; FFM: fat-free mass (total lean tissue plus total bone mineral content); ${ }^{*} P<0.001 ;{ }^{* *} P<0.05$.

TABLE 2: Sex-effect on the proposed cut-off points based on two standard deviations below the mean value of the ASMI and TBSMI derived from healthy young Mexican adults.

\begin{tabular}{lccc}
\hline Variables & Men $(n=136)$ & Women $(n=80)$ & $P$ value \\
\hline ASMI, $\left(\mathrm{kg} / \mathrm{m}^{2}\right)$ & 5.86 & 4.72 & 0.0001 \\
TBSMI, $\left(\mathrm{kg} / \mathrm{m}^{2}\right)$ & 6.63 & 5.22 & 0.0001 \\
\hline
\end{tabular}

ASMI: appendicular skeletal muscle mass index; TBSMI: total-body skeletal muscle mass index.

gender effect was found. As expected, the male group weighed $18 \mathrm{~kg}$ more than the female group $(P<0.001)$, and height in former was higher than in the latter by an average $13 \mathrm{~cm}$ $(P<0.001)$. These differences were reflected significantly in the BMI measurements, and similar results were found for the body composition components assessed. Both, ASM and TBSM were higher in men compared to the mean value for the women's group. These differences were also valid for both indices. However, total and truncal fat were significantly higher in the women group (Table 1).

Table 2 shows the effect of gender on the new proposed cut-off points based on two standard deviations below the mean value of both indices, derived from the sample included. Both indices were significantly higher in the men's group. When these cut-off points were compared to the most often reported indices in the literature, significant differences were found for all indices derived from other sex-specific ethnic groups, except those from a sample of Chinese and Hong Kong men and a sample of Chinese women (Table 3).

\section{Discussion}

This is the first study to use DXA with a sample of young Latin American adults to determine indices based on our skeletal muscle estimates. The cut-off points derived from this sample of male and female Mexican subjects may improve diagnoses of sarcopenia in the Mexican geriatric population or among Latin American people with similar characteristics to those in the sample assessed herein. Overall results confirm the effect of gender on most of the anthropometric and
TABLE 3: Comparison between the proposed sex-specific cut-off points against some published indices from around the world.

\begin{tabular}{lcc}
\hline Indices by authors & \multicolumn{2}{c}{ Sex-specific cut-off points } \\
& Men & Women \\
\hline ASMI, $\left(\mathrm{kg} / \mathrm{m}^{2}\right)$ & & \\
Alemán and Ruiz, 2014 & 5.86 & 4.72 \\
Baumgartner et al., 1988 & $7.26^{*}$ & $5.45^{*}$ \\
Sanada et al., 2010 [7] & $6.87^{*}$ & $5.46^{*}$ \\
Lau et al., 2005 [8] & $5.72^{*}$ & 4.82 \\
Wen et al., 2011 [9] & 5.80 & $4.30^{*}$ \\
Kim et al., 2009 [10] & $7.40^{*}$ & $5.10^{*}$ \\
TBSMI, (kg/m ${ }^{2}$ ) & & \\
Alemán and Ruiz, 2014 & 6.71 & 5.22 \\
Lau et al., 2005 [8] & $7.66^{*}$ & $6.40^{*}$ \\
Tichet et al., 2008 [11] & $8.60^{*}$ & $6.20^{*}$ \\
Chien et al., 2008 [12] & $8.90^{*}$ & $6.50^{*}$ \\
Mesanes et al., 2012 & $8.31^{*}$ & $6.68^{*}$ \\
\hline
\end{tabular}

ASMI: appendicular skeletal muscle mass index; TBSMI: total-body skeletal muscle mass index.

${ }^{*} P<0.001$.

body composition components and DXA-derived indices. In addition, our results showed significant differences with most of the sex-specific population indices published. These results emphasize that these published indices cannot be used interchangeably.

The expected results on the differences between the derived indices from this sample and those published previously can be related to the determinants of differences in body composition, especially skeletal muscle. It is well recognized that skeletal muscle depends on age, gender, ethnicity, body weight, height, and longer extremities $[3,20$, 21]; therefore skeletal muscle indices could depend on the same factors. In order to assess the clinical impact of those indices physical performance test should be assess in order to look the association between the sex-specific cut-off points and physical disabilities in different ethnic groups, but we 
cannot ignore the effect of the techniques used to measure muscle mass since its highly likely that all these determinants may influence the indices.

The indices derived from some Caucasian populations are higher (i.e., Baumgarnert et al.s cut-off points of 7.26 and 5.45 for young man and women adults, resp., [6]) because, in general, Caucasian people are taller than Latina American or Asian populations, while in the case of the African-American people we find significantly greater skeletal muscle mass [20] and it has been reported longer appendicular bone lengths $[22,23]$ compared to Caucasian subjects. If we compared the African-Americans with other ethnic groups, the results would probably not change. For all these reasons, plus the high prevalence of loss of skeletal muscle and sarcopenia around the world, and the clinical impact of these conditions, more research is needed to obtain good reference studies that will establish valid SM cut-off points for populations worldwide and to translate this information to the clinical practice in order to detect patients with low muscle mass or sarcopenia.

The clinical implication of the results of this study is that the interchangeably use of these cut-off points may under- or overestimate real prevalence of loss of skeletal muscle mass or sarcopenia in older people in Mexico or other Latin American countries. Specifically, if we were to use the cut-off points reported by Baumgartner et al., a significant underestimation of the prevalence of the loss of skeletal muscle mass or sarcopenia would be reported for older people, characterized by low skeletal muscle mass or ASMI [20]. Similarly, after applying the ASMI criteria for sarcopenia in a study of an elderly Chinese population Wen et al. [9] concluded that this method may not be appropriate for diagnosing sarcopenia in that group because no older adults in the study were diagnosed with this condition. The criteria based on the skeletal muscle index proposed by Janssen et al. [24] showed greater discriminating power in identifying persons with low handgrip strength. Considering the findings from Wen et al. [9] in addition to having sex-specific cut-off points for different populations or ethnic groups it is clear that the use of other criteria for sarcopenia and clinical assessments of functionality is required for target population.

With respect to clinical implications of the results of this study mentioned before, it is important to consider that skeletal muscle is a required component for diagnoses of sarcopenia [5]. Therefore, the cut-off points for muscle strength in the young reference population or low physical performance in elderly participant in addition to the sexspecific cut-off points for skeletal muscle may improve diagnosis of sarcopenia in the target elderly population.

4.1. Strengths and Limitations. The strength of this study rests on the use of DXA to assess lean tissue, since this technique accurately measures ASM. The comparison with other cutoff points based on DXA-derived ASM is another strength, although the use of a nonrandom sampling method and the nonpopulation-based nature of this work limit the ability to generalize results. For these reasons, future populationbased studies are needed to obtain better reference cut-off points for ASM and other body composition components in the young Mexican adult population. Also, we are aware that the reported cut-off points based on ASM or TBSM derived from a young adults population adjusted to the dimensions of the DXA bed could be another important limitation, as individuals taller than 1.96 meters could not be assessed by DXA. The estimates of TBSMI using a published DXA-equation derived from a Caucasian population and the absence of a gold standard method to assess TBSM could be an additional limitation.

\section{Conclusion}

In conclusion, this study confirms an effect of sex on several body composition components and DXA-derived indices such as ASMI and TBSMI in the sample of young adult assessed. Published cut-off points from Caucasians are higher than those derived in this sample of men and women Mexican subjects so the use of those published indices interchangeably could underestimate the prevalence of the loss of skeletal muscle mass or sarcopenia in older people characterized by low skeletal muscle mass or ASMI. The new DXA-derived cut-off points for ASM proposed herein may improve diagnoses of sarcopenia in the geriatric Mexican population or that of other Latin American countries with similar characteristics to those assessed in our study sample.

\section{Conflict of Interests}

The authors declare that they have no conflict of interests regarding the publication of this paper.

\section{Authors' Contributions}

H. Alemán-Mateo was the main author of the paper and project director and participated in concept, design, data collection, data analysis, data interpretation, and writing and Roxana E. Ruiz Valenzuela participated in data collection, data analysis, and data interpretation.

\section{Acknowledgments}

The authors thank Centro de Investigación en Alimentación y Desarrollo, and CONACYT (S0008-2010-1-140157) for the funding provided for this phase of the study. The authors are especially grateful to the volunteers for their unconditional participation in the project and also Ana Cristina Gallegos for her technical assistance.

\section{References}

[1] I. Janssen and R. Ross, "Linking age-related changes in skeletal muscle mass and composition with metabolism and disease," Journal of Nutrition, Health and Aging, vol. 9, no. 6, pp. 408-419, 2005.

[2] S. P. Tzankoff and A. H. Norris, "Effect of muscle mass decrease on age-related BMR changes," Journal of Applied Physiology Respiratory Environmental and Exercise Physiology, vol. 43, no. 6, pp. 1001-1006, 1977. 
[3] D. Gallagher, M. Visser, R. E. De Meersman et al., "Appendicular skeletal muscle mass: effects of age, gender, and ethnicity," Journal of Applied Physiology, vol. 83, no. 1, pp. 229-239, 1997.

[4] I. Janssen, S. B. Heymsfield, Z. Wang, and R. Ross, "Skeletal muscle mass and distribution in 468 men and women aged 1888 yr," Journal of Applied Physiology, vol. 89, no. 1, pp. 81-88, 2000.

[5] A. J. Cruz-Jentoft, J. P. Baeyens, J. M. Bauer et al., "Sarcopenia: European consensus on definition and diagnosis," Age and Ageing, vol. 39, no. 4, pp. 412-423, 2010.

[6] R. N. Baumgartner, K. M. Koehler, D. Gallagher et al., "Epidemiology of sarcopenia among the elderly in New Mexico," American Journal of Epidemiology, vol. 147, no. 8, pp. 755-763, 1998.

[7] K. Sanada, M. Miyachi, M. Tanimoto et al., "A cross-sectional study of sarcopenia in Japanese men and women: reference values and association with cardiovascular risk factors," European Journal of Applied Physiology, vol. 110, no. 1, pp. 57-65, 2010.

[8] E. M. C. Lau, H. S. H. Lynn, J. W. Woo, T. C. Y. Kwok, and L. J. Melton III, "Prevalence of and risk factors for sarcopenia in elderly Chinese men and women," Journals of Gerontology A, vol. 60, no. 2, pp. 213-216, 2005.

[9] X. Wen, M. Wang, C.-M. Jiang, and Y.-M. Zhang, "Are current definitions of sarcopenia applicable for older Chinese adults?" Journal of Nutrition, Health and Aging, vol. 15, no. 10, pp. 847851, 2011.

[10] T. N. Kim, S. J. Yang, H. J. Yoo et al., "Prevalence of sarcopenia and sarcopenic obesity in Korean adults: the Korean sarcopenic obesity study," International Journal of Obesity, vol. 33, no. 8, pp. 885-892, 2009.

[11] J. Tichet, S. Vol, D. Goxe, A. Salle, G. Berrut, and P. Ritz, "Prevalence of sarcopenia in the French senior population," Journal of Nutrition, Health and Aging, vol. 12, no. 3, pp. 202206, 2008.

[12] M.-Y. Chien, T.-Y. Huang, and Y.-T. Wu, "Prevalence of sarcopenia estimated using a bioelectrical impedance analysis prediction equation in community-dwelling elderly people in Taiwan," Journal of the American Geriatrics Society, vol. 56, no. 9, pp. 1710-1715, 2008.

[13] F. Masanes Toran, A. Culla, M. Navarro-Gonzalez et al., "Prevalence of sarcopenia in healthy community-dwelling elderly in an urban area of Barcelona (Spain)," Journal of Nutrition, Health and Aging, vol. 16, no. 2, pp. 184-187, 2012.

[14] M. C. Velázquez Alva, M. E. Irigoyen Camacho, J. Delgadillo Velázquez, and I. Lazarevich, "The relationship between sarcopenia, undernutrition, physical mobility and basic activities of daily living in a group of elderly women of Mexico city," Nutrición Hospitalaria, vol. 28, no. 2, pp. 514-521, 2013.

[15] A. Y. Bijlsma, C. G. M. Meskers, C. H. Y. Ling et al., "Defining sarcopenia: the impact of different diagnostic criteria on the prevalence of sarcopenia in a large middle aged cohort," Age, vol. 35, no. 3, pp. 871-881, 2013.

[16] World Health Organisation, Obesity: Preventing and Managing the Global Epidemic: Report of the WHO Consultation of Obesity, World Health Organization, Geneva, Switzerland, 1997.

[17] S. B. Heymsfield, R. Smith, M. Aulet et al., "Appendicular skeletal muscle mass: measurement by dual-photon absorptiometry," American Journal of Clinical Nutrition, vol. 52, no. 2, pp. 214-218, 1990.

[18] R. D. Hansen, C. Raja, A. Aslani, R. C. Smith, and B. J. Allen, "Determination of skeletal muscle and fat-free mass by nuclear and dual- energy X-ray absorptiometry methods in men and women aged 51-84 y," American Journal of Clinical Nutrition, vol. 70, no. 2, pp. 228-233, 1999.

[19] J. Kim, Z. Wang, S. B. Heymsfield, R. N. Baumgartner, and D. Gallagher, "Total-body skeletal muscle mass: estimation by a new dual-energy X-ray absorptiometry method," American Journal of Clinical Nutrition, vol. 76, no. 2, pp. 378-383, 2002.

[20] H. Aleman-Mateo, S. Y. Lee, F. Javed et al., "Elderly Mexicans have less muscle and greater total and truncal fat compared to African-Americans and Caucasians with the same BMI," Journal of Nutrition, Health and Aging, vol. 13, no. 10, pp. 919923, 2009.

[21] A. M. Silva, W. Shen, M. Heo et al., "Ethnicity-related skeletal muscle differences across the lifespan," American Journal of Human Biology, vol. 22, no. 1, pp. 76-82, 2010.

[22] L. Gerace, A. Aliprantis, M. Russell et al., "Skeletal differences between black and white men and their relevance to body composition estimates," American Journal of Human Biology, vol. 6, no. 2, pp. 255-262, 2005.

[23] R. M. Malina, "Regional body composition: age, sex, and ethnic variation," in Human Body Composition, A. F. Roche, S. B. Heymsfield, and T. G. Lohman, Eds., pp. 217-255, Human Kinetics, Champaign, Ill, USA, 1996.

[24] I. Janssen, S. B. Heymsfield, and R. Ross, "Low relative skeletal muscle mass (sarcopenia) in older persons is associated with functional impairment and physical disability," Journal of the American Geriatrics Society, vol. 50, no. 5, pp. 889-896, 2002. 


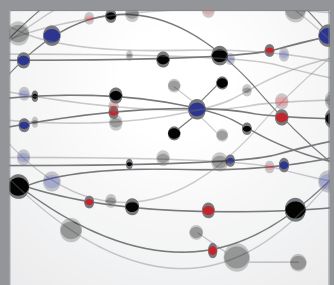

The Scientific World Journal
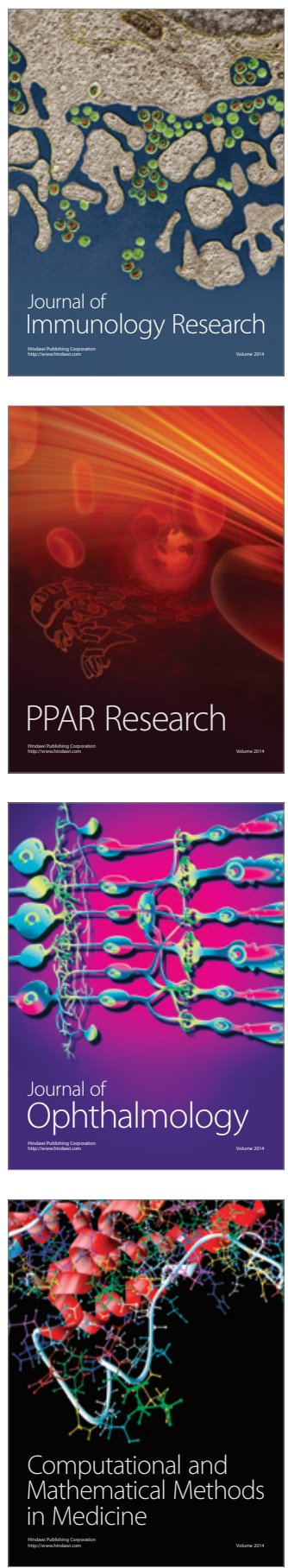

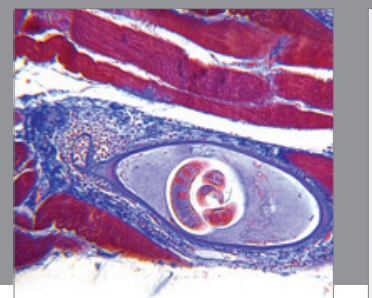

Gastroenterology

Research and Practice
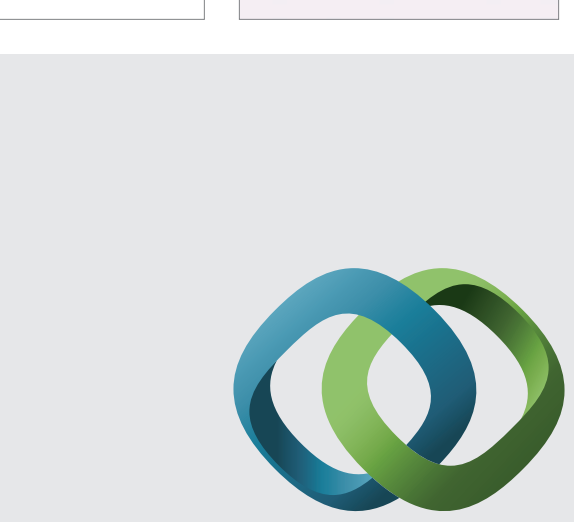

\section{Hindawi}

Submit your manuscripts at

http://www.hindawi.com
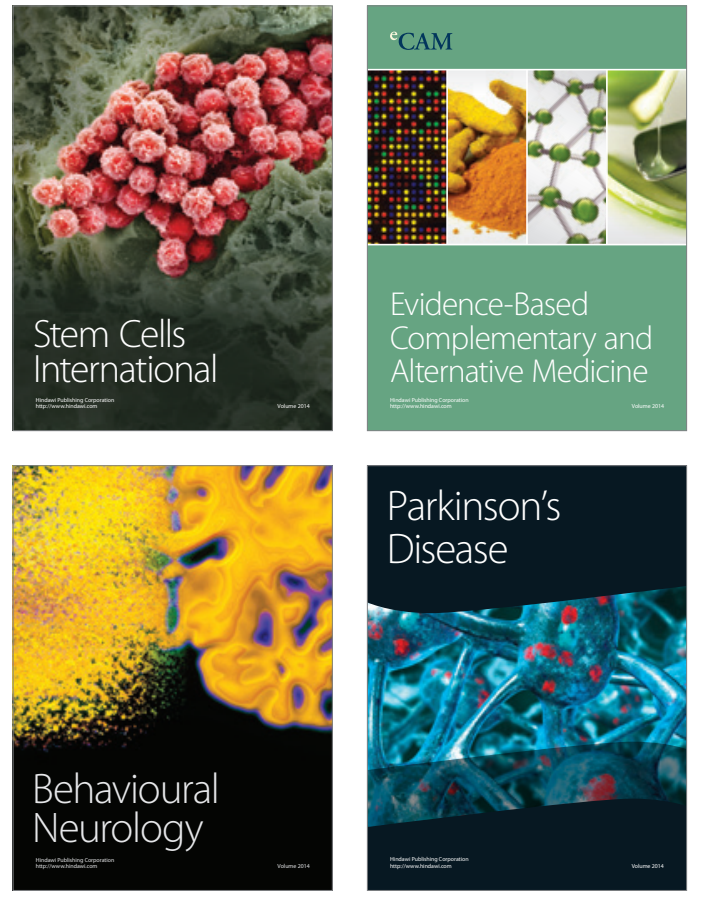
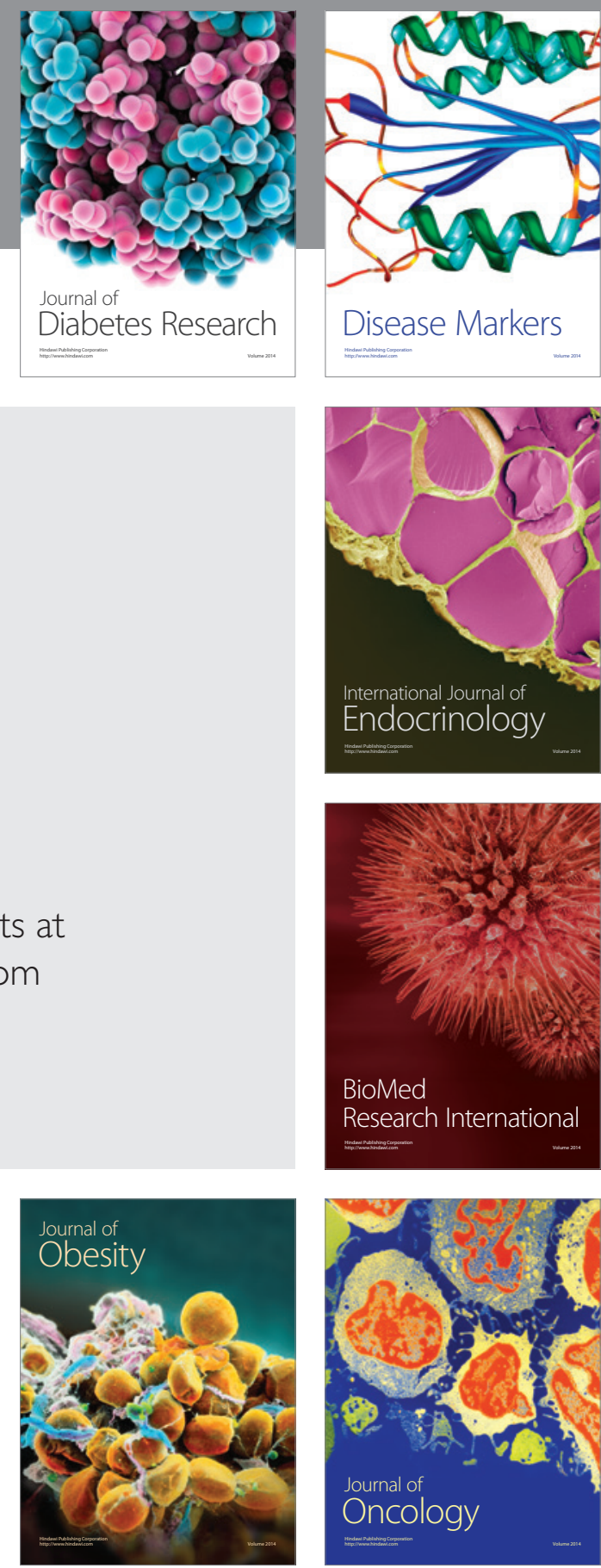

Disease Markers
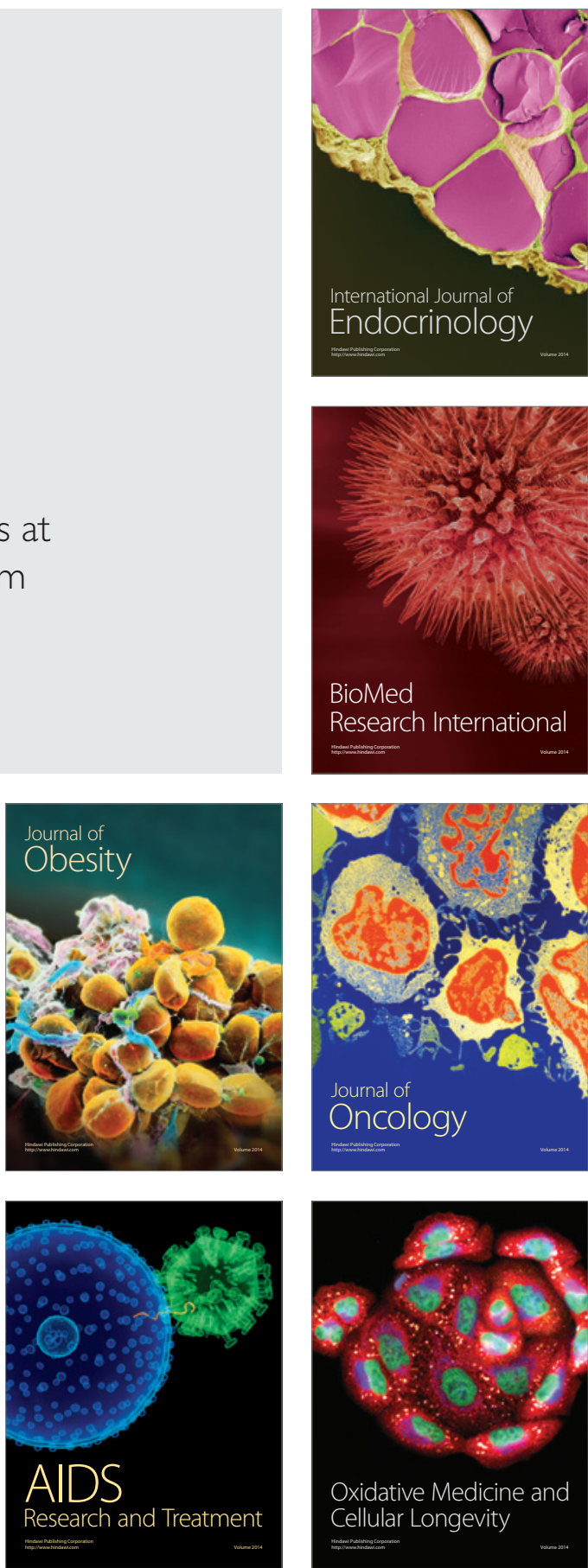